\title{
Entanglement versus relaxation and decoherence in a quantum algorithm for quantum chaos
}

\author{
S. Bettelli, and D. L. Shepelyansky * \\ Laboratoire de Physique Quantique, UMR 5626 du CNRS, \\ Université Paul Sabatier, 31062 Toulouse Cedex 4, France
}

(Dated: July 2, 2018)

\begin{abstract}
We study analytically and numerically the behavior of the concurrence (a measure of the entanglement of formation) of a pair of qubits in a quantum computer operating an efficient algorithm for quantum chaos. Our results show that in an ideal algorithm the entanglement decays exponentially with the diffusive relaxation rate induced by classical chaos. This decay reaches a residual level which drops exponentially with the number of qubits $n_{q}$. Decoherence destroys the residual entanglement with a rate exponential in $n_{q}$.

PACS numbers: 03.67.Lx, 05.45.Mt
\end{abstract}

Enormous interest into quantum information and computation [1] has generated serious efforts in characterizing and understanding quantum entanglement which is considered as the ultimate origin of quantum power (see a recent review 2]). A quantitative measure of the entanglement of formation, namely the concurrence $C$, was introduced and shown to be able to characterize an arbitrary state of two qubits [3, 4]. Being closely related to the von Neumann entropy $S$ of the reduced density matrix $\rho$ of two qubits 31], this quantity was recently found to have interesting applications to quantum phase transitions in interacting spin systems [5]. In parallel, the properties of entanglement were investigated in a quantum model of coupled tops where it was shown that there exists a typical value of entanglement which is determined by the chaotic behavior of the dynamics of the model 6] and that the growth rate of entanglement of initially decoupled tops is increased by the underlying classical chaos [7]. On the same line, it was recently shown that contrary to intuition even a heat bath may create entanglement between two qubits [8].

All these studies [5, 6, 7, 8] clearly demonstrated how rich entanglement properties can be in interacting quantum systems. However, in the context of quantum computation it is much more crucial to analyze the evolution of entanglement in a specific algorithm performing an operational task. Indeed, it is expected that the entanglement is very sensitive to noise and decoherence 9, 10, 11. and the understanding of its behavior in an operating algorithm can lead to better strategies in the control of decoherence and imperfection effects. As for our knowledge such direct investigations have not been performed until now. Therefore, in this paper we study the behavior of the concurrence in an efficient algorithm for the quantum sawtooth map which has been proposed recently in [12]. The algorithm for this model has a number of important advantages: all $n_{q}$ qubits are used in an optimal way and no ancillae are required, one map iteration in

${ }^{*} \mathrm{URL}:$ http://www.quantware.ups-tlse.fr the Hilbert space of size $N=2^{n_{q}}$ is performed in $O\left(n_{q}^{2}\right)$ quantum gates, and the algorithm is based on the quantum Fourier transform (QFT) which is one of the main elements of various quantum algorithms 1]. This allows to simulate a complex dynamics in the regime of quantum chaos with a small number of qubits. Since the entanglement can be efficiently measured experimentally (see e.g. 13]) the experimental observation of the concurrence behavior discussed here can be realized on NMR [14, 15] or ion-trap [16] based quantum computers with about 6 10 qubits and a few hundreds of gates.

Contrary to the situation discussed in $[6,7]$, our results show that in the exact quantum algorithm the underlying classical chaos leads to an exponential decrease of the concurrence $C$ down to some residual level $\bar{C}$ which characterizes the global system coherence. On the other hand, the presence of noise in the quantum gates leads to a destruction of this coherence with a rate $\Gamma$ growing exponentially with the number of qubits $n_{q}$. This shows that entanglement can be very sensitive to decoherence.

The dynamics of the classical sawtooth map [17, 18] is given by:

$$
\bar{n}=n-k \frac{d V(\theta)}{d \theta}, \quad \bar{\theta}=\theta+T \bar{n} \bmod 2 \pi
$$

where $V(\theta)=-\theta^{2} / 2,-p i \leq \theta<\pi$ and the bars denote the variables after one iteration. After rescaling $y=T n$ and $x=\theta$, it is clear that the dynamics depends only on the parameter $K=k T$. Due to the discontinuity in the derivative of $V(\theta)$, the Kolmogorov-Arnold-Moser (KAM) theorem cannot be applied to the map (1) and its dynamics becomes chaotic and diffusive for arbitrarily small values of the chaos parameter $K>0$ [17]. For $K \ll 1$ the diffusion is governed by a nontrivial cantori regime which has been worked out in [17]. In this case the rescaled diffusion rate $D_{0}(K)=(\Delta y)^{2} / t \approx 1.2 \pi^{2} K^{2.5} / 3$ is much smaller than the quasi-linear diffusion rate corresponding to the random phase approximation $D_{q l}=$ $\pi^{2} K^{2} / 3$ (the latter becomes valid only at $K \gg 1$ ). The diffusion rate in $n$ is $D=D_{0}(K) / T^{2}$.

The quantum sawtooth map $12,19,20,21,22]$ is the quantized version of the classical map, to which it corre- 


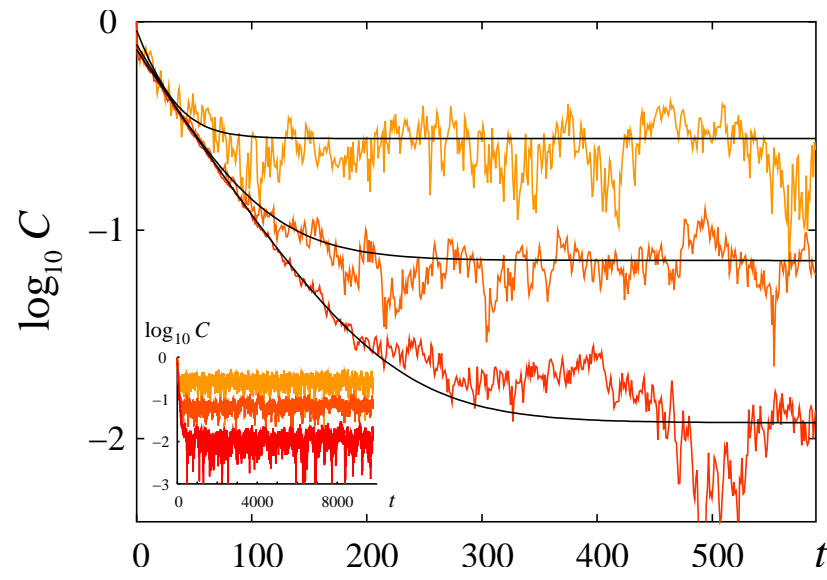

FIG. 1: Dependence of the concurrence $C$ on the dimensionless time $t$ (the number of map iterations) for the map (2) at $K=0.5, L=4$ and $n_{q}=8,12,16$ (curves from top to bottom respectively). The smooth curves show the fit $C(t)=A \exp (-\gamma t)+\bar{C}$ of the relaxation to the asymptotic value $\bar{C}$ obtained on a larger time interval $\left(t \leq 10^{4}\right)$. The inset shows $C(t)$ on larger time scale. The initial state is $(|00\rangle+|11\rangle)|\phi\rangle / \sqrt{2}$ where $|\phi\rangle$ is the uniform superposition of all but the two most significant qubits. Here and below the logarithms are decimal and all axis units are dimensionless.

sponds in the limit $k \rightarrow \infty, T \rightarrow 0$, and $K=k T=$ const. One step in the quantum map is given by the unitary operator $U$ acting on the wave function $\psi(\theta)$ :

$$
\overline{\psi(\theta)}=\hat{U} \psi(\theta)=e^{i k \theta^{2} / 2} e^{-i T \hat{n}^{2} / 2} \psi(\theta),
$$

where $\hat{n}=-i \partial / \partial \theta$ (we set $\hbar=1$ ); when $n_{q}$ qubits are used for the discretization, so that the total number of levels is $N=2^{n_{q}}$, we assume periodic boundary conditions on a torus in the phase representation $(\psi(\theta+2 \pi)=\psi(\theta))$ and in the momentum representation $(\psi(n+N)=\psi(n))$, as discussed in [12, 23]. As a result, the phase $\theta$ takes only $N=2^{n_{q}}$ discrete equidistant values in the interval $-\pi \leq \theta<\pi$, and so does the momentum $(n=0,1, \ldots, N-1$, i.e. $n+N$ is identified with $n$ ). The sawtooth map approximately describes the dynamics in the stadium billiard [19], the phenomenon of dynamical localization which is similar to the Anderson localization in disordered potentials and cantori induced localization 20, 21, 22]. Thus this simple map describes a rich and complex dynamics and represents an interesting testing ground for efficient quantum computation. It is especially important to understand how this complex quantum dynamics will be affected by imperfections in realistic quantum computers.

The numerical simulation of the map (2) is based on the quantum algorithm described in 12 and is implemented on the basis of a quantum computer language developed in [24] which is well adapted for the experimental operation of quantum gates via classical computer software. In this way the dynamics of up to 20 qubits can be easily simulated on a laptop.

To investigate the behavior of the concurrence in the quantum map (2) we compute $C$ for the two most significant qubits which determine the first two binary digits $a_{1,2}$ in the expansion of momentum $n$ : the reduced density matrix $\rho$ for this qubit pair is obtained by tracing out all other $n_{q}-2$ less significant qubits (the digits $a_{i}$ with $3 \leq i \leq n_{q}$ in the expansion of $\left.n=\left(a_{1} a_{2} a_{3} \ldots a_{i} \ldots a_{n_{q}}\right)\right)$. After that $C$ is computed from $\rho$ as described in [3, 4]. In this way we obtain the concurrence value $C$ on a global scale of the whole system which is decomposed into 4 equal parts with $N / 4$ quantum states in each of them. In addition we fix $T=2 \pi L / N$ in the regime of quantum resonance so that $L$ gives the integer number of classical phase space cells embedded in the quantum torus of size $N$ [12, 23] (the classical dynamics is periodic in $n$ with period $2 \pi / T)$. In the following we also take $L$ to be a multiple of 4 to have an integer number of classical cells in the 4 parts of the partition in the momentum $n$.

Typical examples of the dependence of $C$ on the number of map iterations $t$ are shown in Fig.1. According to these data $C(t)$ decays exponentially down to a residual value $\bar{C}$ and, in the limit of large $N$, the decay rate $\gamma$ becomes independent of $N$. It is natural to compare this rate with the rate of classical relaxation. Indeed, due to underlying classical chaos, the probability distribution $f_{n}=\left|\psi_{n}\right|^{2}$ over $n$ is described by the Fokker-Planck equation $\partial f_{n} / \partial t=D \partial^{2} f_{n} / \partial^{2} n / 2$ which gives the relaxation to equipartition with the rate

$$
\gamma_{c}=2 \pi^{2} D / N^{2}=D_{0}(K) / 2 L^{2} .
$$

The comparison between this classical value $\gamma_{c}$ and the rate of concurrence decay $\gamma$ is given in Fig.2. It clearly shows that the decay rate of $C(t)$ is given by the classical rate: $\gamma=\gamma_{c}$. It is important to stress that this relation remains valid also in the nontrivial cantori regime $(K \ll$ 1 ) and that the quantum decay reproduces all oscillations of the classical diffusion (see inset in Fig.2).

The properties of the residual value of the concurrence $\bar{C}$ are analyzed in Fig.3. We will argue in the following that they can be understood in terms of the system conductance $g(25,26])$; in view of this we express $\bar{C}$ vs. $g=2 \gamma_{c} / \Delta=N D_{0}(K) / L^{2}$ where, up to a constant factor, $\Delta=1 / N$ is the level spacing and $2 \gamma_{c}=D_{0}(K) / L^{2}$ is the Thouless energy (see [27] for a recent review). In spite of strong fluctuations the data presented in Fig. 3 can be described by the global average dependence $\bar{C} \sim 1 / \sqrt{g} \propto 1 / \sqrt{N}$. Indeed, for $K=0.5, L=4$ the system size varies by 3 orders of magnitude and the fit gives an algebraic decay with power $\alpha=0.56 \pm 0.02$ being close to $1 / 2$. We attribute the presence of strong fluctuations to the fact that the value $\bar{C}$ is averaged only over time but there is no averaging over parameters. Thus, from the point of view of disordered systems 27] $\bar{C}$ represents only one value for one realization of disorder.

We propose the following explanation of the results presented in Figs.1-3. For a state $|\psi\rangle$ like in Fig.1, we can write $|\psi\rangle=\sum_{a_{1} a_{2}}\left|a_{1} a_{2}\right\rangle\left|\phi_{a_{1} a_{2}}\right\rangle$ where $a_{1,2}=0$ or 1 . Then the value of the concurrence $C$ is proportional to 


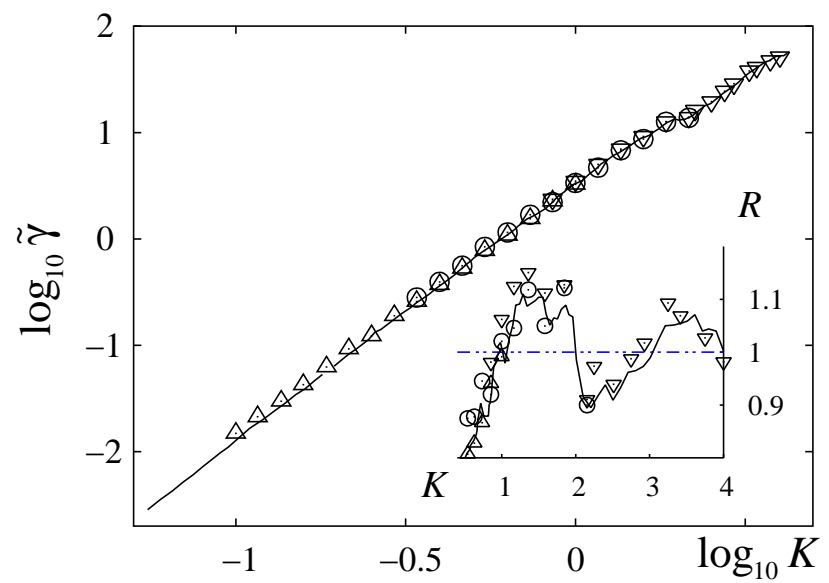

FIG. 2: Dependence of the rescaled rate of the concurrence decay $\tilde{\gamma}=2 \gamma L^{2}$ on the chaos parameter $K$ for $n_{q}=19, L=16$ (triangles down); $n_{q}=18, L=8$ (circles) and $n_{q}=17, L=4$ (triangles up). The solid curve gives the values of the diffusion rate $D_{0}(K)$ taken from Figs.2,3 of 17] showing that $\gamma$ is determined by the classical relaxation rate (3). The inset shows data on a larger scale with $R=\tilde{\gamma} / D_{q l}$ (symbols) and $R=D_{0}(K) / D_{q l}$ (curve from [17]).

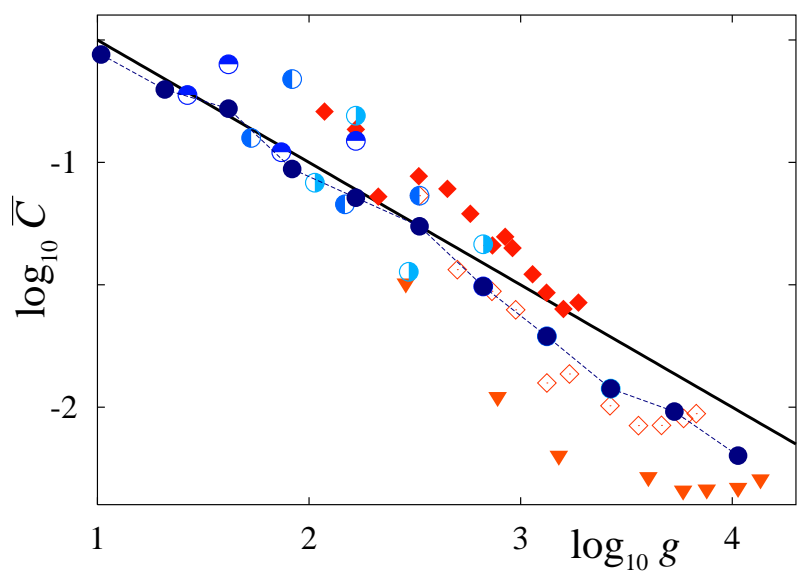

FIG. 3: Dependence of the residual value of the concurrence $\bar{C}$ on the system conductance $g=N D_{0}(K) / L^{2}$ for a broad range of parameters: half filled circles show dependence on $L=4,8,12,16,20$ for $K=0.5$ and $n_{q}=14,15,16$; diamonds and triangles show the variation with $K$ for $n_{q}=14, L=$ $16 ; n_{q}=15, L=8$ and $n_{q}=16, L=4$. The filled circles connected by the dashed curve show the dependence on $N$ for $K=0.5, L=4$. The solid line marks the slope $1 / \sqrt{g}$.

the difference of two scalar products, $C \sim\left|Q_{14}-Q_{23}\right|$, where $Q_{14}=2 \sqrt{\left|\left\langle\phi_{00} \mid \phi_{11}\right\rangle\right|^{2}}$ and $Q_{23}=2 \sqrt{\left|\left\langle\phi_{01} \mid \phi_{10}\right\rangle\right|^{2}}$. From this relation and the fact that the initial state is symmetrically distributed with respect to the transformation $n \rightarrow N-n$, it follows that $C$ is proportional to the difference $\left|W_{11}+W_{00}-W_{01}-W_{10}\right|$ where $W_{a_{1} a_{2}}$ is the total probability inside the part $\left(a_{1} a_{2}\right)$. In the classical limit this probability difference relaxes to zero with the classical relaxation rate $\gamma_{c}$, and that's why $\gamma=\gamma_{c}$ in agreement with the data of Fig.2.

The residual value $\bar{C}$ is determined by the quantum fluctuations of the previous difference of scalar products. In fact, due to the discretization of map (2), the symmetry $n \rightarrow N-n$ is broken and $\left|\phi_{00}\right\rangle$ becomes different from $\left|\phi_{11}\right\rangle$. Therefore in the scalar product $Q_{14}$ (and $\left.Q_{23}\right)$ the $N / 4$ terms have random signs and thus $Q_{14} \propto 1 / \sqrt{N}$ (each term is of the order of $1 / N)$. In this estimate we assumed a summation over all $N$ wave function components. However for finite values of the conductance $g$ only the states inside the Thouless energy interval $2 \gamma_{c}$ have a significant scalar overlap 27, 28], and thus we can make a conjecture that $N$ should be replaced by the effective number of components which is of the order of $N_{\text {eff }} \sim \gamma_{c} / \Delta \sim g$. According to this, $\bar{C} \sim 1 / \sqrt{g}$ in agreement with the data of Fig.3.

The existence of a residual level of concurrence for an ideal quantum algorithm reflects the fact that the global behavior of the whole system remains coherent. In fact, the Poincaré theorem guarantees that for very large times the concurrence will have a revival close to the initial value (however, this will happen on an exponentially large time scale). The situation becomes qualitatively different in presence of external decoherence represented by noisy gates. In our numerical simulations, noisy gates are modeled by unitary rotations by an angle randomly fluctuating in the interval $(-\epsilon / 2, \epsilon / 2)$ around the perfect rotation angle. The presence of this external decoherence leads to a decrease of the residual value of $C$ as illustrated in Fig.4: the constant level is replaced by an exponential decay which gives $\bar{C} \propto \exp (-\Gamma t)$.

In order to obtain the dependence of $\Gamma$ on the parameters we extracted it from the fit of the averaged ratio of $C$ under a noisy evolution to its value in the ideal one. An example of such ratio and the corresponding fit is shown in Fig.4. To suppress fluctuations we averaged over 20 realizations of the noisy evolution. Moreover, the fit was restricted to the plateau regime, where the exact concurrence is fluctuating around its residual value (the initial diffusive relaxation was excluded from the fit).

The results for $\Gamma$ obtained in this way are presented in Fig.5. Quite naturally we find that $\Gamma \propto \epsilon^{2}$, as it was also seen in other simulations of quantum algorithms with noisy gates (e.g. [29]). This scaling becomes better and better for large $\epsilon$ values where $\Gamma$ is larger. However, more surprisingly there is an exponential growth of $\Gamma$ with the number of qubits $n_{q}(\Gamma \propto \sqrt{N})$. This result is very different from those obtained in [12, 29], where the time scale for fidelity and the decoherence rate for tunneling oscillations varied polynomially with $n_{q}$. We see two possible reasons for the exponential sensitivity of the residual concurrence to decoherence. At first, in our case $\Gamma$ is computed over a very large time interval, for which the quantum dynamics already reached its asymptotic behavior (plateau for the residual concurrence); it is known that on very large times the eigenstates are exponentially sensitive to imperfections due to the chaotic structure of the wave functions (see results and discus- 


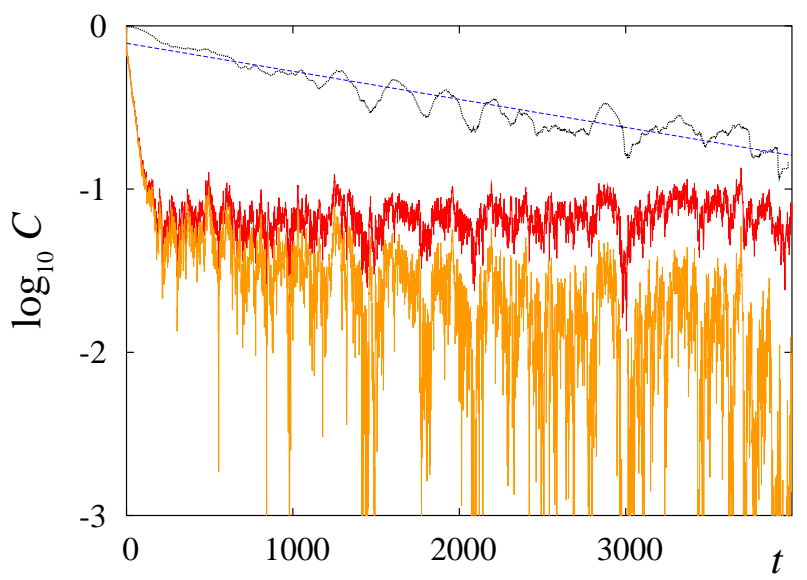

FIG. 4: Effects of decoherence on the residual concurrence: the two lower curves show the dependence $C(t)$ for an ideal algorithm (top) and an algorithm with noisy gates at noise amplitude $\epsilon=0.003$ (bottom). The time $t$ is dimensionless (it is the number of map steps). In the latter case the average is done over 20 noise realizations. The curve in the upper part shows the ratio of $C(t)$ at $\epsilon=0.003$ to its value in an ideal algorithm, this ratio is averaged over a 100-kick moving window to reduce fluctuations. The dashed straight line shows a fit of the ratio to an exponential decay proportional to $e^{-\Gamma t}$. Here $n_{q}=12, K=0.5, L=4$. sions in [30]). Another possible reason can be related to the fact that the residual value of the concurrence on the plateau is on its own exponentially small and maybe this is the reason why it becomes so sensitive to decoherence. Further investigation on the decoherence effects for the concurrence are required to understand in a better way this exponential sensitivity of $C$.

In our studies we restricted ourselves to the investigation of entanglement only between two qubits. The problem of the characterizing the entanglement of a larger number of qubits represents an interesting challenge but at the same time it is much more complicated 2]. However even the relatively simple case of two qubits shows nontrivial links between concurrence and such interesting physical phenomena as quantum phase transitions [5] and statistical relaxation.

In summary, our studies show that the decay of the concurrence in an operating quantum computer is determined by the underlying relaxation rate of the classical dynamics. We show that the residual level of entanglement in an ideal algorithm scales as the inverse square root of the conductance of the system. This residual entanglement is destroyed by decoherence, whose effective rate grows exponentially with the number of qubits.

This work was supported in part by the EC contracts RTN QTRANS and IST-FET EDIQIP and the NSA and ARDA under ARO contract No. DAAD19-01-1-0553.
[1] M. A. Nielsen and I. L. Chuang, Quantum Computation and Quantum Information (Cambridge Univ. Press, Cambridge, 2000).

[2] D. Bruß, J. Math. Phys. 43, 4237 (2002).

[3] S. Hill and W. K. Wootters, Phys. Rev. Lett. 78, 5022 (1997).

[4] W. K. Wootters, Phys. Rev. Lett. 80, 2245 (1998).

[5] A. Osterloh, L. Amico, G. Falci, and R. Fazio, Nature 416, 608 (2002).

[6] P. A. Miller and S. Sarkar, Phys. Rev. E 60, 1542 (1999).

[7] J. N. Bandyopadhyay and A. Lakshminarayan, Phys. Rev. Lett. 89, 060402 (2002).

[8] D. Braun, Phys. Rev. Lett. 89, 277901 (2002).

[9] J. P. Paz and W. H. Zurek, in Coherent Matter Waves, edited by R. Kaiser, C. Westbrook, and F. David (Springer Verlag, Berlin, 2001), pp. 533-614.

[10] R. Schack and C. M. Caves, Phys. Rev. Lett. 71, 525 (1993).

[11] R. Schack and C. M. Caves, Phys. Rev. E 53, 3257 (1996).

[12] G. Benenti, G. Casati, S. Montangero, and D. L. Shepelyansky, Phys. Rev. Lett. 87, 227901 (2001).

[13] P. Horodecki and A. Ekert, Phys. Rev. Lett. 89, 127902 (2002).

[14] Y. S. Weinstein, M. A. Pravia, E. M. Fortunato, S. Lloyd, and D. G. Cory, Phys. Rev. Lett. 86, 1889 (2001).

[15] L. M. K. Vandersypen, M. Steffen, G. Breyta, C. S. Yannoni, M. H. Sherwood, and I. L. Chuang, Nature 414, 883 (2001).
[16] S. Gulde, M. Riebe, G. P. T. Lancaster, C. Becher, J. Eschner, H. Häffner, F. Schmidt-Kaler, I. L. Chuang, and R. Blatt, Nature 421, 48 (2003).

[17] I. Dana, N. W. Murray, and I. C. Percival, Phys. Rev. Lett. 62, 233 (1989).

[18] Q. Chen, I. Dana, J. D. Meiss, N. W. Murray, and I. C. Percival, Physica D 46, 217 (1990).

[19] F. .Borgonovi, G. Casati, and B. Li, Phys. Rev. Lett. 77, 4744 (1996).

[20] F. Borgonovi, Phys. Rev. Lett. 80, 4653 (1998).

[21] G. Casati and T. Prosen, Phys. Rev. E 59, R2516 (1999).

[22] R. E. Prange, R. Narevich, and O. Zaitsev, Phys. Rev. E 59, 1694 (1999).

[23] F. M. Izrailev, Phys. Rep. 196, 299 (1990).

[24] S. Bettelli, L. Serafini, and T. Calarco (2003), to appear in Eur. Phys. J. D, cs.PL/0103009.

[25] J. T. Edwards and D. J. Thouless, J. Phys. C 5, 807 (1972).

[26] D. J. Thouless, Phys. Rep. 13, 93 (1974).

[27] A. D. Mirlin, Phys. Rep. 326, 259 (2000).

[28] Y. M. Blanter, Phys. Rev. B 54, 12807 (1996).

[29] A. D. Chepelianskii and D. L. Shepelyansky, Phys. Rev. A 66, 054301 (2002).

[30] G. Benenti, G. Casati, S. Montangero, and D. L. Shepelyansky, Eur. Phys. J. D 20, 293 (2002).

[31] Given a density matrix $\rho$ for a pair of two-level systems, the concurrence $C$ is defined as $\min \left\{0, \lambda_{1}-\lambda_{2}-\lambda_{3}-\lambda_{4}\right\}$, where the $\lambda_{i}$ are the eigenvalues, in decreasing value order, of the hermitian matrix $\sqrt{\sqrt{\rho} \widetilde{\rho} \sqrt{\rho}}$, with $\widetilde{\rho}=$ 


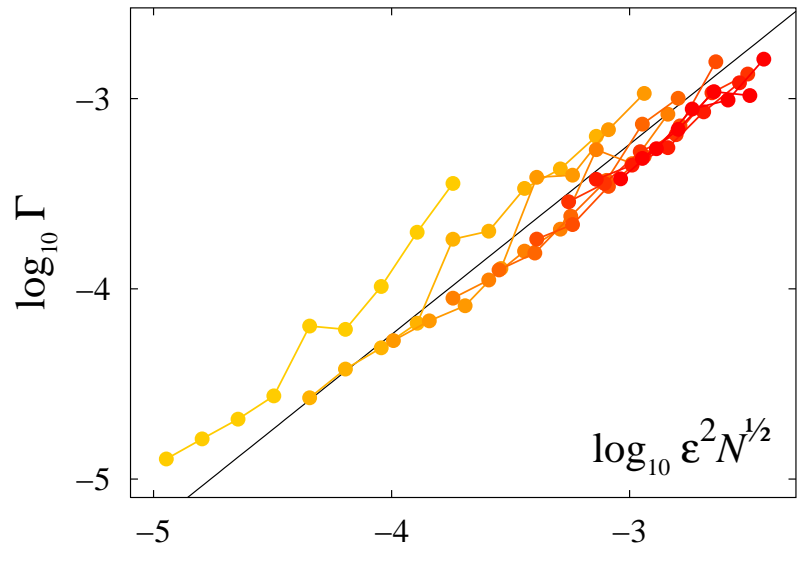

FIG. 5: Dependence of the decoherence induced decay rate $\Gamma$ of the residual concurrence on $\epsilon^{2} \sqrt{N}$ for $K=0.5, L=$ 4. Here the noise amplitude $\epsilon$ changes from 0.001 to 0.01 (10 equidistant values) for $7 \leq n_{q} \leq 15$. The data points (circles) are connected by lines for fixed values of $\epsilon$. The color intensity changes gradually from one chain to another to mark the variation of $\epsilon$ (low/high intensity corresponds to small/large values of $\epsilon$ ). The straight line shows the averaged behavior $\Gamma=0.58 \epsilon^{2} \sqrt{N}$. $\left(\sigma_{y} \otimes \sigma_{y}\right) \rho^{*}\left(\sigma_{y} \otimes \sigma_{y}\right)$. The von Neumann entropy $S$ of $\rho$ monotonically increases from 0 to 1 when $C$ goes from 0 to 1 [3, 4]. 